\section{Homing in on homeogenes}

Identifying the target genes of homeoprotein transcription factors is important for understanding the genetic networks underlying development, and pathologies that arise from mutations in these loci. Prochiantz and colleagues have developed a gene trap screen for systematically identifying DNA elements that bind to homeoproteins. The gene trap was integrated into a library of embryonic stem (ES) cells, which placed lac $Z$ under control of the trapped gene. When the DNA-binding portion of a homeoprotein-the homeoboxis applied to the cells exogenously, it crosses the cell membrane and enters the nucleus, where it interferes with the repressor function of endogenous homeoproteins, as revealed by LacZ staining. Because the third helix of homeodomains can efficiently transport other peptides or compounds to the nucleus, the strategy potentially can be applied to other types of transcriptional regulation (see p. 746).

$N D$

\section{Engineering toxin-gobbling bacterium}

The widespread industrial use of tetrachloroethylene (PCE) makes it one of the five most common groundwater pollutants, one that is both toxic and a suspected carcinogen. Until now, however, PCE had been thought to be impossible to biodegrade aerobically. Extrapolating from the ability of a purified soluble methane monooxygenase to degrade chlorofluoroethylene aerobically, Ryoo et al. have evaluated the ability of a monooxygenase from the wastewater bacterium Pseudomonas stutzeri OX1 to oxidize PCE. They found that toluene-o-xylene monooxygenase (ToMO), expressed by whole $P$. stutzeri OX1 cells, efficiently degraded PCE by converting it to free chloride ions. Because ToMO has also been shown to degrade simultaneously mixtures of other chlorinated aliphatics, this enzyme offers great potential in cleaning up the mixtures of PCE and its less chlorinated degradation products commonly found at many hazardous waste sites (see p. 775).

This Month in Nature Biotechnology written by Natalie DeWitt, Judy Jamison, Andrew Marshall, and Meeghan Sinclair.

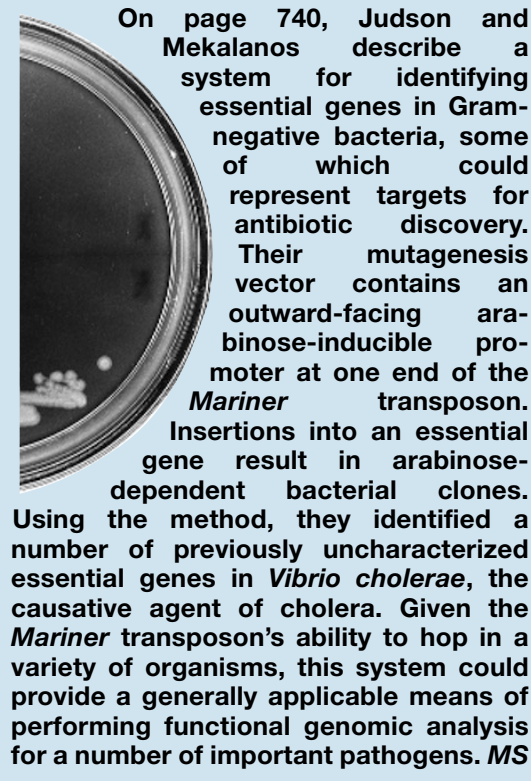

\section{A paper boon?}

Traditional methods of breeding desirable traits in trees have been hampered by their slow generation times. Reasoning that increasing biosynthesis of gibberellin, an important plant hormone, might be an effective way of changing tree growth and development processes, Eriksson and coworkers have transformed aspen trees with an Arabidopsis gene encoding GA 20oxidase, a key enzyme in gibberellin biosynthesis that is normally downregulated by the end-product gibberellins. The transgenic trees not only grew faster in both height and diameter, they had more numerous and longer xylem fibers and increased biomassall traits that could be a boon to the pulp and paper industries (see p. 784).

Lupus is characterized by pathogenic autoantibodies directed against cellular components, resulting in diverse symptoms of the disease, including arthritis, anemia, and central nervous system and kidney damage. These autoantibodies interact with Fc-receptor $\gamma$-chains $(F c \gamma R)$, triggering an inflammatory response typical of the disease. On page 735 , Marino et al. describe development and testing of a tetrameric tripeptide that can bind to the constant region of IgGs and interfere with IgG-Fc $\gamma R$ interaction in vitro. In a murine model used to study lupus, administration of the peptide enhanced survival and prevented immune complex formation in the kidney without apparent signs of toxicity.

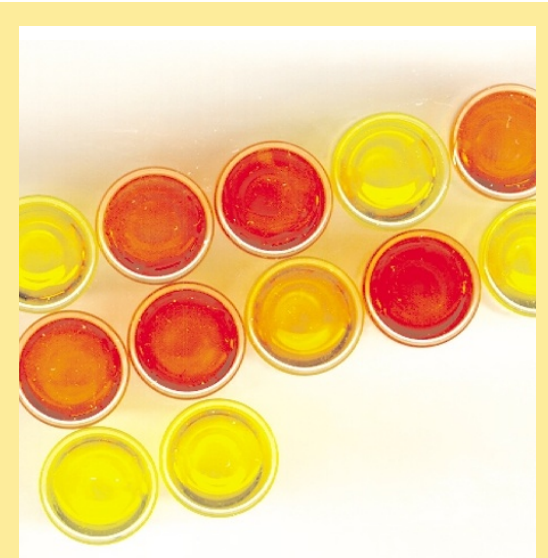

On page 750 , Schmidt-Dannert et al. demonstrate that we need not be content with the chemical structures nature has wrought. Using a new twist on molecular evolution, they created chimeric genes that catalyze formation of carotenoids never before produced by conventional metabolic engineering of Escherichia coli. Instead of limiting their starting genes to related genes from one species, they shuffle carotenoid biosynthetic genes from several different bacterial species. Using this approach, they recombined the genes encoding phytoene desaturase and lycopene cyclase, and then visually screened the resulting bacterial colonies for coloration reflecting different double bonding or cyclization of the products catalyzed by the mutant enzymes. Such molecular breeding of biosynthetic pathways could potentially yield new biologically active compounds with relatively little effort.

ND

\section{The importance of being three-dimensional}

Methods for generating $\mathrm{T}$ cells in vitro from human $\mathrm{CD} 34^{+}$typically have been cumbersome and yielded few mature $\mathrm{T}$ cells. Now on page 729, Poznansky et al. have used a biocompatible three-dimensional carbon matrix to engineer an artificial thymus for T-cell production. First, they seeded matrices with thymic tissue from mice, then they added human bone marrow-derived progenitor cells to the mix. In this environment, the progenitor cells produced large quantities of functional $\mathrm{T}$ cells, as shown by a number of assays, including proliferative response to mitogen, cytokine production, and HIV-1 infectability. Interestingly, they found that the matrix architecture was key, confirming the importance of three-dimensional cell-cell interactions for successful tissue engineering (See also p. 714). 